\title{
Decreased Expression of Sirt1 Contributes to Ocular Behçet's Disease Progression via Th17 and Th22 Response
}

\author{
Manyun Xie ${ }^{a, b}$ Yan Yang ${ }^{a, b}$ \\ aDepartment of Ophthalmology, The Second Xiangya Hospital of Central South University, Changsha, China; \\ ${ }^{b}$ Hunan Clinical Research Center of Ophthalmic Disease, Changsha, China
}

\section{Keywords}

Sirt1 - Ocular Behçet's disease · Th17 cells · Th22 cells

\begin{abstract}
Background: Previous studies have indicated that Sirtuin 1 (Sirt1) plays an important role in suppressing inflammatory responses in many diseases. However, the Sirt1 levels and role of Sirt 1 in ocular Behçet's disease (OBD) have not been fully elucidated. Objective: The objective of this study was to investigate the role of Sirt1 in the pathogenesis of OBD. Methods: Sirt1 and cytokine levels were measured using ELISA. Cell viability was determined using the Cell Counting Kit-8. The frequencies of Th17 and Th22 cells were detected using flow cytometry. Results: We found decreased expression of Sirt 1 in $\mathrm{CD}^{+} \mathrm{T}$ cells obtained from patients with active OBD. SRT1720, an agonist of Sirt1, significantly upregulated Sirt 1 expression in CD4 ${ }^{+}$T cells from patients with active OBD. Sirt1 activation by SRT1720 significantly suppressed the production of interleukin (IL)-17 and IL-22 by $\mathrm{CD}^{+}{ }^{+}$T cells and inhibited the expansion of Th17 and Th22 cells. Conclusion: Our results suggest that decreased Sirt1 expression might be involved in the pathogenesis of OBD and that activation of Sirt1 might be considered a potential target for OBD.

(c) 2020 S. Karger AG, Basel
\end{abstract}

\section{Introduction}

Behçet's disease (BD) is a multi-systemic autoimmune disease that is characterized by recurrent uveitis, oral and genital ulceration, and multiple skin lesions, and ocular $\mathrm{BD}(\mathrm{OBD})$ is one of the most prevalent forms of uveitis in China [1]. Autoinflammatory pathways are presumed to play an important role in its pathogenesis [2]. A previous study focusing on the interleukin (IL)-23/IL-17 pathway in $\mathrm{BD}$ found the frequency of IL-17-producing $\mathrm{CD} 4^{+} \mathrm{T}$ cells was significantly higher than IL-17-producing $\mathrm{CD} 8^{+}$ T cells in patients with active OBD [3]. Cai et al. [4] found that the frequency of IL-22-producing $\mathrm{CD} 4^{+} \mathrm{T}$ cells was higher in patients with active $\mathrm{BD}$, but there was no difference in IL-22-producing $\mathrm{CD}^{+} \mathrm{T}$ cells. Based on these previous studies, $\mathrm{CD} 4^{+} \mathrm{T}$ cells play an essential role in the development of autoimmune diseases. Therefore, in this study, we focused on these 2 subsets of Th cells, Th17 and Th22 cells.

Sirtuin 1 (Sirt1) is a class III histone deacetylase that deacetylates histone and non-histone proteins [5]. Initially, Sirtl was reported to be involved in various biological processes, such as aging, metabolism, and development $[6,7]$. Recent studies have demonstrated a key role for Sirt 1 in T cell immunity and T cell-related autoimmune

Correspondence to:

Yan Yang, yangyan5973@csu.edu.cn 
Table 1. Demographics of the enrolled BD patients and controls

\begin{tabular}{|c|c|c|c|c|c|c|}
\hline & \multicolumn{2}{|l|}{ Ba patients } & \multicolumn{2}{|l|}{ Bi patients } & \multicolumn{2}{|c|}{ Normal controls } \\
\hline & $n($ total $=20)$ & $\%$ & $n($ total $=8)$ & $\%$ & $n($ total $=24)$ & $\%$ \\
\hline Age, mean, years & 37.2 & & 36.4 & & 36.8 & \\
\hline Male & 16 & 80 & 6 & 75 & 19 & 79.2 \\
\hline Female & 4 & 20 & 2 & 25 & 5 & 20.8 \\
\hline
\end{tabular}

$\mathrm{Ba}$, active $\mathrm{OBD} ; \mathrm{Bi}$, inactive $\mathrm{OBD}$. diseases. It has been reported that the expression of Sirt1 was higher in anergic T cells [8]. Knockdown of SIRT1 leads to increased release of cytokines [9]. Moreover, SIRT1-knockout mice exhibit an autoimmune-like condition with loss of peripheral tolerance and enhancement of T-cell activation and proliferation $[8,10]$.

In view of the effect of Sirt 1 on T cells and the involvement of this protein in inflammatory conditions, in this study, we investigated whether Sirt 1 is involved in the pathogenesis of OBD. Our results show that decreased Sirtl expression was associated with OBD activity and that it can inhibit the expansion and cytokine secretion of both Th17 and Th22 cells.

\section{Materials and Methods}

\section{Patients and Healthy Controls}

For this study, we enrolled 20 patients with active OBD (defined as $\mathrm{Ba}, 16$ men and 4 women, average age: 37.2 years) and 8 patients with $\mathrm{BD}$ without active ocular inflammation 3 months after treatment with corticosteroids and immunosuppressants (defined as $\mathrm{Bi}, 6$ men and 2 women, average age: 36.4 years), who were admitted to the First Affiliated Hospital of Chongqing Medical University and the Second Xiangya Hospital of Central South University from March 2018 to March 2019. Healthy individuals $(n=24)$ served as controls (CNs). The diagnosis of BD was made according to the criteria of the International Study Group for BD [11]. The distribution of demographic characteristics of the included patients with BD and healthy controls is shown in Table 1. There was no difference in age or sex distribution. All Ba patients showed active intraocular inflammation. Active intraocular inflammation was defined largely depending on the presence of dust keratic precipitates, anterior chamber cells, vitreous cells, and retinal vasculitis observed clinically or revealed by fluorescein angiography. The extraocular manifestations of these Ba patients were recurrent oral ulceration (100\%), recurrent genital ulceration (52\%), and skin lesions (72.8\%). This study was approved by the Ethics Committee of the First Affiliated Hospital of Chongqing Medical University (approval reference number: 2018-048) and the Second Xiangya Hospital of Central South University (approval reference number: 2016-079). All procedures followed the tenets of the Declaration of Helsinki, and written informed consent was obtained from all patients and healthy controls.

\section{Cell Isolation and Culture}

Anticoagulated whole blood samples were obtained from patients with active OBD and healthy controls. Peripheral blood mononuclear cells (PBMCs) were isolated using density gradient centrifugation (Ficoll-Hypaque; TBDScience, Tianjin, China). $\mathrm{CD} 4^{+} \mathrm{T}$ cells were isolated using human CD4 microbeads followed by magnetic-activated cell sorting according to the manufacturer's instructions (Miltenyi Biotec, Palo Alto, CA, USA). PBMCs and $\mathrm{CD}^{+} \mathrm{T}$ cells were resuspended at $1 \times 10^{6}$ cells/mL in RPMI 1640 medium (Gibco; Invitrogen, Carlsbad, CA, USA) containing Lglutamine $(2 \mathrm{mM})$, penicillin/streptomycin $(100 \mathrm{U} / \mathrm{mL})$, and $10 \%$ fetal calf serum (Gibco; Invitrogen, Carlsbad, CA, USA), and cells were cultured with SRT1720 $(10 \mathrm{nM}-10 \mu \mathrm{M})$ or DMSO vehicle; T cell activation was promoted in the presence of anti-CD3 $(0.5 \mu \mathrm{g} /$ $\mathrm{mL}$ ) and anti-CD28 antibodies $(0.1 \mu \mathrm{g} / \mathrm{mL}$ ) (Miltenyi Biotec, Palo Alto, CA, USA).

\section{Cell Viability Detection}

$\mathrm{CD}^{+} \mathrm{T}$ cells were seeded in 96-well plates and cultured with different concentrations of SRT1720 for $24 \mathrm{~h}$. Cell Counting Kit-8 (KeyGEN BioTECH, Jiangsu, China) solution was added to each well and incubated for another $3 \mathrm{~h}$. The absorbance was determined at $450 \mathrm{nM}$ using a Varioskan LUX Multimode Reader (Thermo Fisher Scientific, Inc.). Viability was calculated using the following equation: cell viability $(\%)=(\mathrm{OD}$ treatment/OD control) $\times 100$.

\section{Flow Cytometry}

$\mathrm{CD}^{+} \mathrm{T}$ cells were cultured with SRT1720 or DMSO vehicle in the presence of anti-CD3 $(0.5 \mu \mathrm{g} / \mathrm{mL})$ and anti-CD28 antibodies $(0.1 \mu \mathrm{g} / \mathrm{mL})$. For Th17 and Th22 cell frequencies, the cells were stimulated with a Cell Activation Cocktail (with Brefeldin A) (BioLegend, San Diego, CA, USA) according to the manufacturer's protocol. The stimulated cells were fixed, permeabilized, and stained with anti-IL-17A, anti-IL-22 antibodies, or isotypes (eBioscience, San Diego, CA, USA). Flow cytometry analysis was performed using a FACS Aria cytometer (BD Biosciences, San Diego, CA, USA) and analyzed using FlowJo software (Tree Star, Inc., San Carlos, CA, USA).

\section{Enzyme-Linked Immunosorbent Assay}

Sirt1 in the PBMCs and CD4 ${ }^{+} \mathrm{T}$ cells was quantified using an Abcam SimpleStep ELISA kit (ab171573). IL-17 and IL-22 levels in the supernatants from $\mathrm{CD} 4^{+} \mathrm{T}$ cells were measured using DuoSet ELISA kits (R\&D Systems, Minneapolis, MN, USA). 


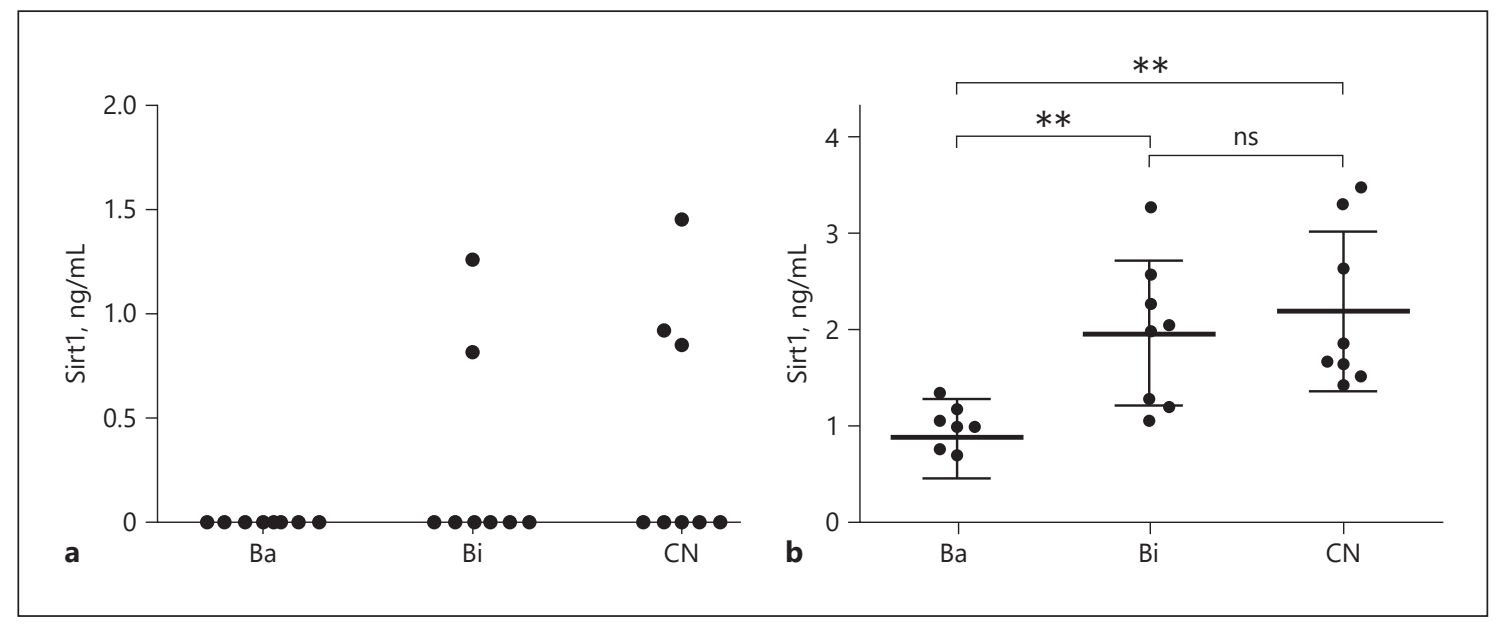

Fig. 1. Decreased expression of Sirt1 in PBMCs and CD4 ${ }^{+} \mathrm{T}$ cells obtained patients with from Ba. a ELISA for Sirt1 expression in PBMCs $(n=8)$. b ELISA for Sirt1 expression in CD4 ${ }^{+}$T cells $(n=8, t$ test or Mann-Whitney $\mathrm{U}$ test, ${ }^{* *} p<0.01$, ns $p \geq 0.05$ ). Ba, active OBD; Bi, inactive OBD; CN, normal control; PBMC, peripheral blood mononuclear cell; ns, nonsignificant.

Statistical Analysis

All data are presented as mean $\pm \mathrm{SD}$. The Shapiro-Wilk normality test was used to assess normality. One-way ANOVA, $t$ test, and Mann-Whitney U test were performed using Prism 5 software (GraphPad Software, USA). Nonsignificant differences are denoted as ns. The $p$ values are denoted as follows: ${ }^{*} p<0.05,{ }^{* *} p<0.01$, and ${ }^{* * *} p<0.001$.

\section{Results}

\section{Decreased Sirt1 Expression in Patients with Active $O B D$}

To study whether Sirtl plays a role in the pathogenesis of OBD, we first compared the expression of Sirt1 in PBMCs between patients with active OBD and healthy controls. The levels of Sirt1, except for 3 samples from healthy controls, were below the detectable limit of the ELISA kit (less than $0.625 \mathrm{ng} / \mathrm{mL}$ ). We further tested the Sirt1 expression from enriched $\mathrm{CD} 4^{+} \mathrm{T}$ cells, and the levels of Sirt 1 were found to be significantly lower in CD $4^{+}$ $\mathrm{T}$ cells obtained from patients with active OBD $(0.87 \pm$ $0.40 \mathrm{ng} / \mathrm{mL}$ ) than those from patients with inactive $\mathrm{BD}(1.96 \pm 0.76 \mathrm{ng} / \mathrm{mL}, p<0.01)$ and healthy controls $(2.19 \pm 0.83 \mathrm{ng} / \mathrm{mL}, p<0.01)$. However, there was no difference between patients with inactive BD and healthy controls (Fig. 1).

\section{Effect of SRT1720 on Cell Viability of $\mathrm{CD}^{+}$T Cells}

To study the role of Sirt1 in inflammatory cytokine secretion by $\mathrm{CD} 4{ }^{+} \mathrm{T}$ cells, we first tested whether SRT1720,

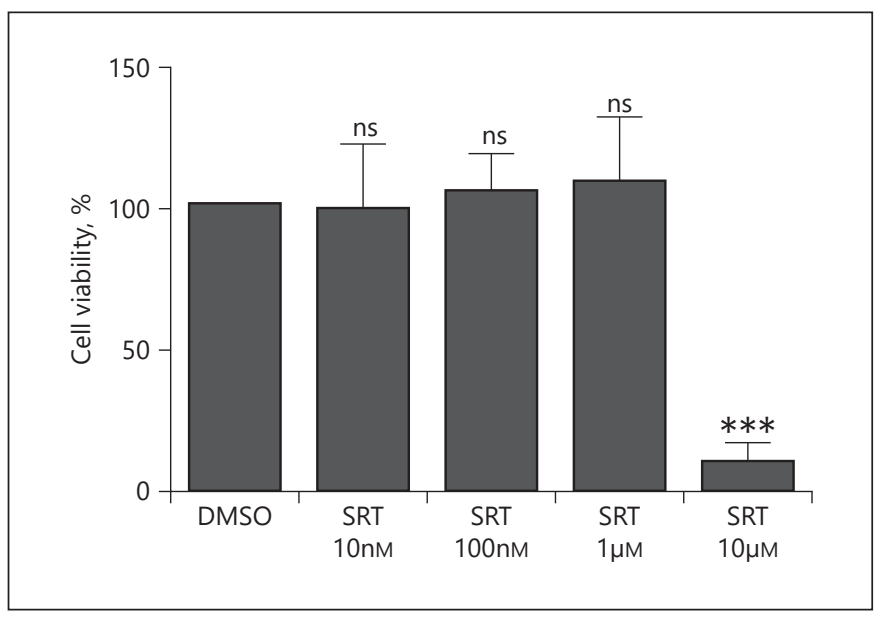

Fig. 2. Cell viability was measured in $\mathrm{CD} 4^{+} \mathrm{T}$ cells from healthy donors in the presence of increasing concentrations of SRT1720 ( $n=8$, one-way ANOVA and Bonferroni's multiple comparison test, ${ }^{* * *} p<0.001$, ns $\left.p \geq 0.05\right)$. SRT, SRT1720.

a specific agonist of SIRT1, had an effect on the viability of $\mathrm{CD} 4^{+} \mathrm{T}$ cells. $\mathrm{CD} 4^{+} \mathrm{T}$ cells were cultured using different concentrations of SRT1720 $(0-10 \mu \mathrm{M})$ in the presence of anti-CD3 and anti-CD28 antibodies for 3 days to detect possible cytotoxicity. SRT1720 had no effect on the viability of $\mathrm{CD} 4^{+} \mathrm{T}$ cells up to a concentration of $1 \mu \mathrm{M}$, whereas higher concentrations $(10 \mu \mathrm{M})$ resulted in cell death (Fig. 2). Finally, a concentration of $1 \mu \mathrm{M}$ was used in subsequent experiments. 


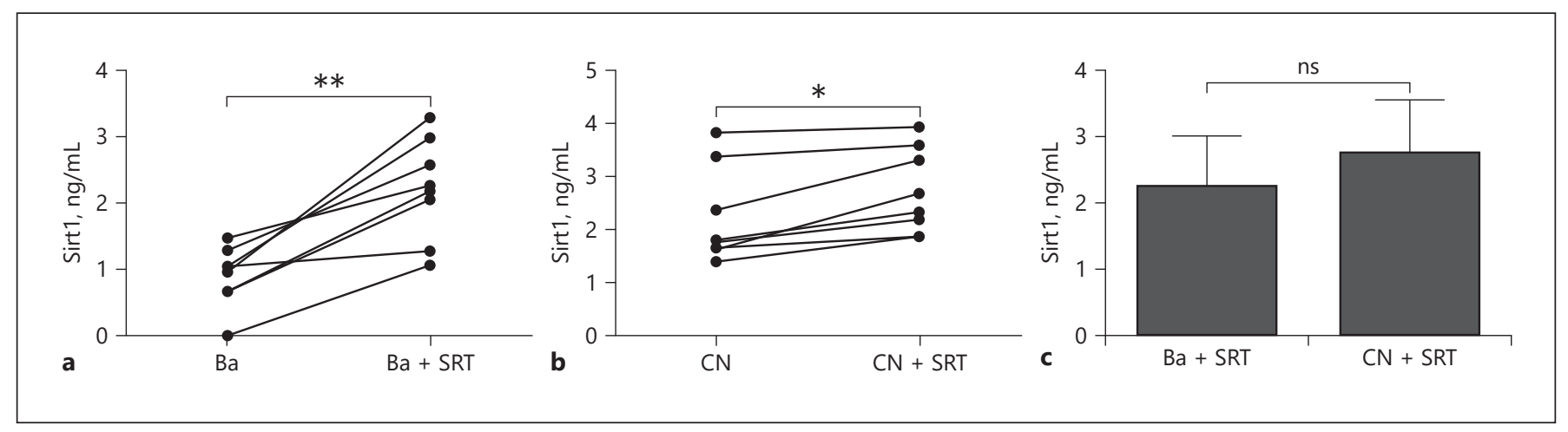

Fig. 3. Sirt 1 expression in CD4 ${ }^{+} \mathrm{T}$ cells was restored by SRT1720 activation. a Sirt1 expression in CD4 ${ }^{+} \mathrm{T}$ cells from Ba. b Sirt1 expression in CD4 ${ }^{+}$T cells from normal controls. c Sirt1 levels in SRT1720 treated groups $(n=$ $8, t$ test or Mann-Whitney U test, ${ }^{* *} p \leq 0.01$, ns $\left.p \geq 0.05\right)$. Ba, active OBD; Bi, inactive OBD; CN, normal controls; SRT, SRT1720 $1 \mu \mathrm{M}$.

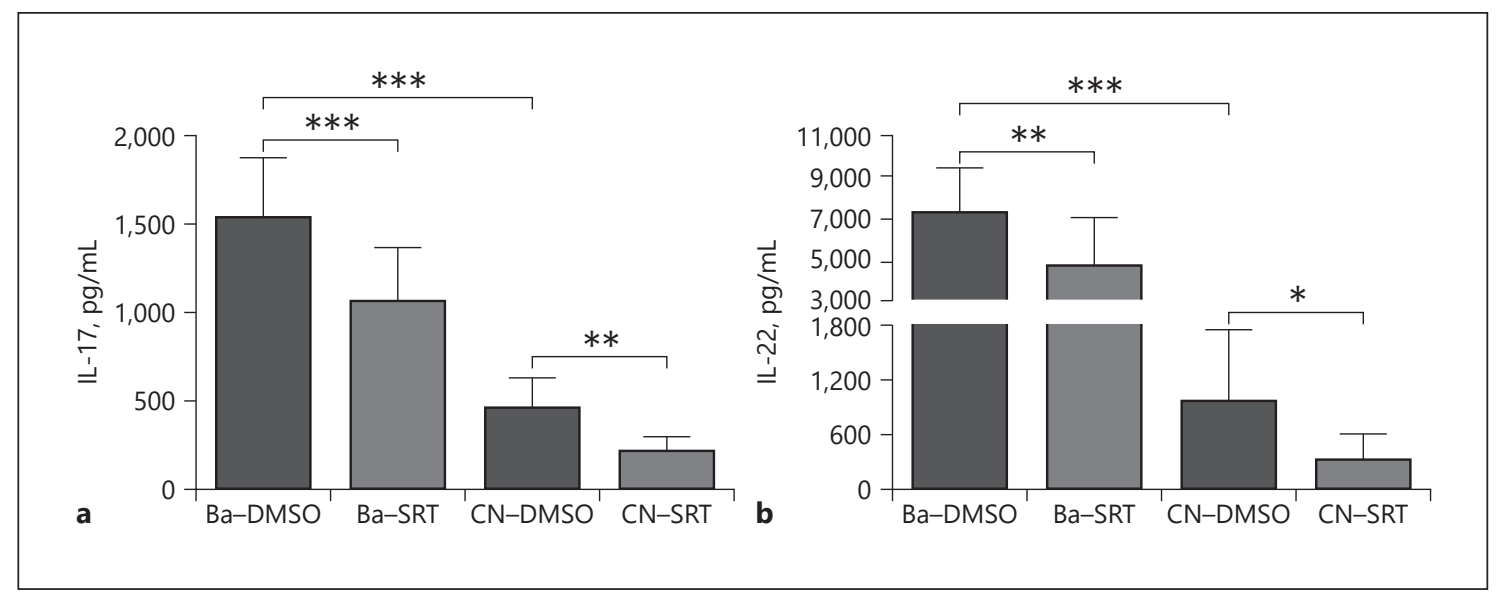

Fig. 4. Effect of SRT1720 on the IL-17 and IL-22 production by CD4 ${ }^{+} \mathrm{T}$ cells. a IL-17 expression by CD4 ${ }^{+} \mathrm{T}$ cells obtained from $\mathrm{Ba}$ and normal controls. $\mathbf{b}$ IL-22 expression by $\mathrm{CD} 4^{+} \mathrm{T}$ cells obtained from $\mathrm{Ba}$ and normal controls $\left(n=8, t\right.$ test or Mann-Whitney U test, $\left.{ }^{*} p<0.05,{ }^{* *} p<0.01,{ }^{* * *} p<0.001\right)$. Ba, active OBD; Bi, inactive OBD; CN, normal controls; SRT, SRT1720 $1 \mu \mathrm{M}$.

Sirt1 Expression Was Restored by SRT1720 Activation Does SRT1720 affect Sirt 1 expression in CD4 ${ }^{+}$T cells? After coculturing with or without SRT1720 $(1 \mu \mathrm{M})$ for 24 $\mathrm{h}$, Sirt1 expression was upregulated 2.5 -fold in the active OBD patient group, but only 1.3 -fold in the control group (Fig. 3a, b). In the SRT1720 cocultured groups, although Sirtl expression was a still little lower in the active OBD group than in the control group $(2.21 \pm 0.76$ vs. $2.71 \pm$ $0.80 \mathrm{ng} / \mathrm{mL}, p>0.05)$, there was no significant difference (Fig. 3c).

\section{Sirt1 Activation Suppressed Inflammatory Cytokine} Secretion by $\mathrm{CD}^{+}$T Cells

We subsequently investigated the effect of Sirt1 activation on cytokine secretion by $\mathrm{CD} 4^{+} \mathrm{T}$ cells. The data showed that IL-17 and IL-22 levels were significantly lower after SRT1720 stimulation. These results indicate that the increased expression of IL-17 and IL-22 was at least partly due to Sirt1 reduction (Fig. 4).

Influence of Sirt1 Activation on the Expansion of Th17 and Th22 Cells

Purified $\mathrm{CD}^{+} \mathrm{T}$ cells were treated with or without SRT1720 to evaluate Sirt1 activation on Th17 and Th22 cell expansion. The results showed that the percentage of 


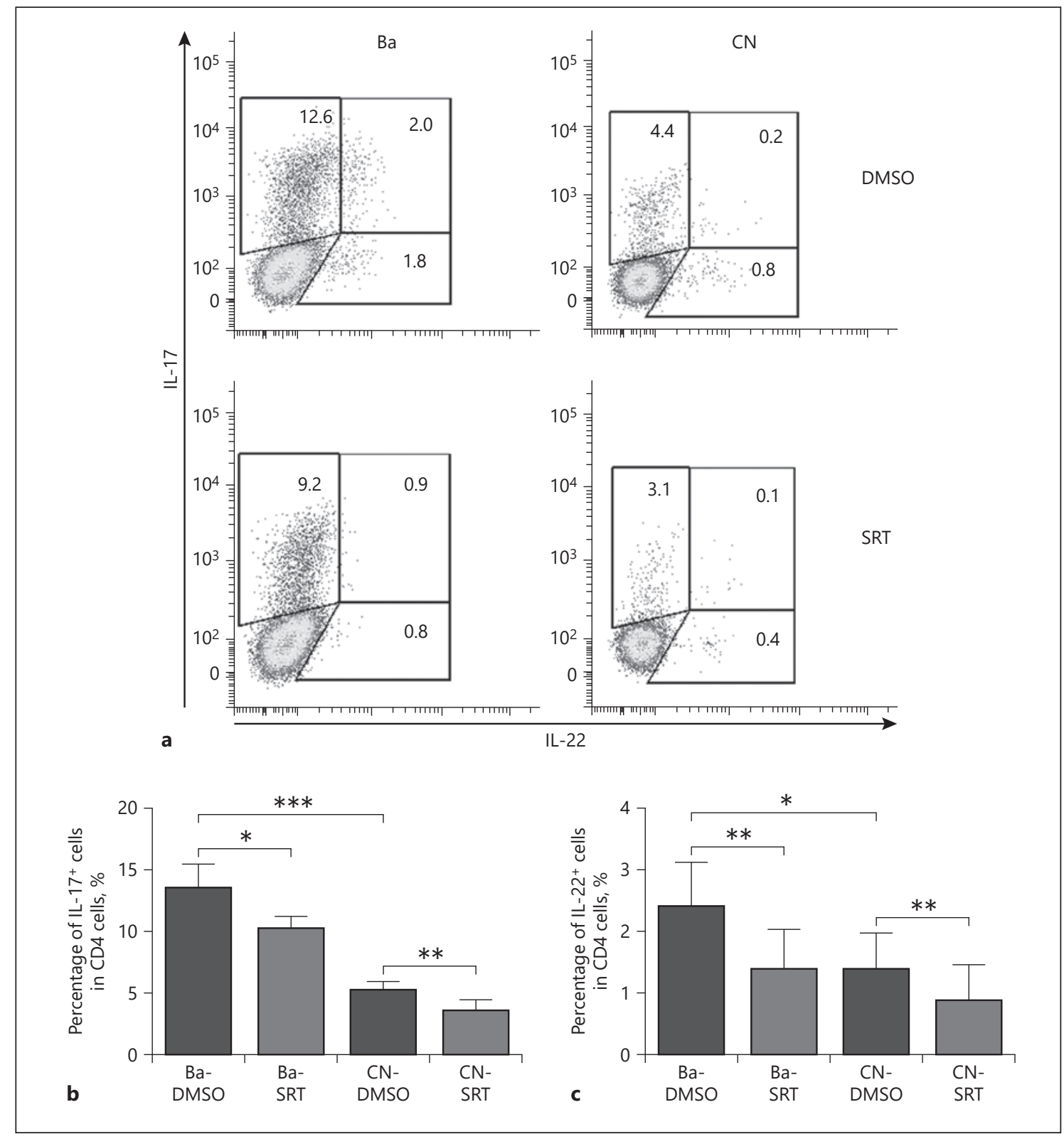

Fig. 5. Effect of SRT1720 on Th17 and Th22 expansion. a Representative plots of IL-17 ${ }^{+}$cells, IL-22 $2^{+}$cells, IL- $17^{+} \mathrm{IL}-22^{+}$cells in an $\mathrm{Ba}$ and a normal control. $\mathbf{b}$ The results represent the percentages of IL-17 ${ }^{+}$cells among $\mathrm{CD}^{+} \mathrm{T}$ cells obtained from $\mathrm{Ba}$ and normal controls. $\mathbf{c}$ The results represent the percentages of IL-22 $2^{+}$cells among $\mathrm{CD}^{+}{ }^{+} \mathrm{T}$ cells obtained from $\mathrm{Ba}$ and normal controls $\left(n=6, t\right.$ test or Mann-Whitney $\mathrm{U}$ test, ${ }^{*} p<0.05,{ }^{* *} p<0.01$, $* * * p<0.001)$. Ba, active OBD; Bi, inactive OBD; CN, normal controls; SRT, SRT1720 $1 \mu \mathrm{M}$.

Th17 and Th22 cells was significantly higher in patients with active OBD than in healthy controls (Th17 cells: $13.43 \pm 2.08 \%$ vs. $5.19 \pm 0.72 \%, p<0.001$; Th22 cells: $2.40 \pm 0.71 \%$ vs. $1.38 \pm 0.58 \%, p<0.05)$ in the absence of SRT1720. The addition of SRT1720 significantly decreased the expansion of Th17 and Th22 cells in both active OBD patients and healthy controls (Fig. 5).

\section{Discussion}

In the present study, we investigated the expression and function of Sirtl in the pathogenesis of OBD. The results showed that Sirt1 expression in both PBMCs and $\mathrm{CD} 4^{+} \mathrm{T}$ cells from active OBD patients was markedly lower than that in normal controls, suggesting that Sirt1 
may participate in the pathogenesis of OBD. Sirt1 activation with the agonist SRT1720 restored the expression of Sirt1. Further studies showed that Sirt1 activation could inhibit Th17- and Th22-related pro-inflammatory cytokine secretion in $\mathrm{CD} 4^{+} \mathrm{T}$ cells and inhibit the expansion of Th17 and Th22 cells.

The anti-inflammatory role of Sirt1 has been reported in many inflammatory conditions $[9,10,12]$. However, literature on the expression and function of Sirt1 in Th cells in OBD is limited. Gardner and his colleagues reported that activation of Sirt1 resulted in suppressed proliferation of $\mathrm{CD}^{+} \mathrm{T}$ cells in $\mathrm{OBD}$ patients and proinflammatory cytokine production [13], while the expression of Sirt 1 and the effect of Th17 and Th22 cells require further study. For this purpose, we first estimated the Sirt1 levels in PBMCs and $\mathrm{CD}^{+}{ }^{+} \mathrm{T}$ cells in patients with OBD. This result was in disagreement with a study performed in patients with systemic lupus erythematosus, which reported increased Sirt1 levels in $\mathrm{CD} 4^{+} \mathrm{T}$ cells in patients with active systemic lupus erythematosus [14]. However, our results are generally consistent with those of earlier studies on rheumatoid arthritis, diabetes, and chronic obstructive pulmonary disorder [15-17]. This result suggests that downregulated Sirt 1 expression was associated with inflammation in OBD patients.

Our study showed that Sirt1 activation with SRT1720 could restore protein expression of Sirt 1 in $\mathrm{CD}^{+} \mathrm{T}$ cells in OBD patients, which is generally consistent with an earlier study performed in normal CD $4^{+} \mathrm{T}$ cells [18]. Sirt1 activation acts as a positive transcriptional regulator by enhancing transcription factor activity, resulting in elevated Sirtl protein expression $[19,20]$.

Previous studies have demonstrated that Th17 and Th22 cells play an important role in the pathogenesis of OBD, while IL-17 and IL-22 are representative cytokines of Th17 and Th22 cells. An earlier study demonstrated that IL-17 levels were elevated in OBD [21]. Two independent studies reported high levels of IL-22 and IL-22+ Th cells in BD patients $[4,21]$. In our study, we also demonstrated that IL-17 and IL-22 levels were elevated in active OBD patients, and Sirtl could inhibit the secretion of IL-17 and IL-22. Furthermore, we tested whether Sirt1 activation had an effect on Th17 and Th22 cell expansion. Previous studies performed in animal models showed that Sirt1 could promote Th17 differentiation directly or indirectly, and treatment with a Sirt 1 inhibitor could suppress the development of experimental autoimmune encephalomyelitis [22, 23]. However, in experimental autoimmune uveoretinitis, an animal model for human uve- itis, Sirt 1 activation suppressed intraocular inflammation [13]. Our results are generally in line with the latter study. The results show that Sirt 1 activation could also inhibit the expansion of Th17 and Th2 2 cells, and the inhibitory effect did not depend on the influence of cell viability. Therefore, we inferred that Sirt1 participated in the pathogenesis of OBD by affecting the secretion of proinflammatory cytokines and the expansion of Th17 and Th22 cells.

Why Sirt 1 is decreased in the $\mathrm{CD} 4^{+} \mathrm{T}$ cells of patients with active OBD remains unclear. Since the expression of Sirtl decreases with advancing age in several tissues [24, 25], there was no difference between patients and healthy controls with respect to age. Previous studies showed that cigarette smoke extract causes a decrease in Sirt1 levels in monocyte-macrophage cells $[26,27]$, and ultraviolet B could also decrease the expression of Sirt 1 in $\mathrm{CD} 4^{+} \mathrm{T}$ cells [18]. These studies suggested that environmental factors had an effect on Sirt1 expression. Further studies concerning the possible factors that influence Sirt1 levels in patients with OBD need to be studied. In summary, in this study, we observed decreased expression of Sirt 1 in active $\mathrm{OBD}$, and further experiments suggest that this decrease might affect the Th17 and Th22 response, leading to the development of OBD.

\section{Acknowledgements}

We would like to thank Professor Peizeng Yang for providing the samples in the study. We would also like to thank all patients and healthy donors enrolled in the present study.

\section{Statement of Ethics}

This study was approved by the Ethics Committee of the First Affiliated Hospital of Chongqing Medical University (approval reference number: 2018-048) and the Second Xiangya Hospital of Central South University (approval reference number: 2016-079). All procedures followed the tenets of the Declaration of Helsinki, and written consent was obtained from all patients and healthy controls. We would like to thank Editage (www.editage.cn) for English language editing.

\section{Conflict of Interest Statement}

There are no conflicts of interest. 


\section{Funding Sources}

This work was supported by the National Natural Science Foundation of China (No. 81600724).

\section{Author Contributions}

Manyun Xie conducted the experiments and analyzed the data. Yan Yang designed the experiment, conducted the experiments, analyzed the data, and wrote the manuscript.

\section{References}

1 Yang P, Fang W, Meng Q, Ren Y, Xing L, Kijlstra A. Clinical features of chinese patients with Behçet's disease. Ophthalmology. 2008; 115(2):312-318.e4.

2 Khairallah M, Accorinti M, Muccioli C, Kahloun R, Kempen JH. Epidemiology of Behçet disease. Ocul Immunol Inflamm. 2012; 20(5):324-35.

3 Chi W, Zhu X, Yang P, Liu X, Lin X, Zhou H, et al. Upregulated IL-23 and IL-17 in Behçet patients with active uveitis. Invest Ophthalmol Vis Sci. 2008;49(7):3058-64.

4 Cai T, Wang Q, Zhou Q, Wang C, Hou S, Qi $\mathrm{J}$, et al. Increased expression of IL-22 is associated with disease activity in Behcet's disease. PLoS One. 2013;8(3):e59009.

5 Kong S, McBurney MW, Fang D. Sirtuin 1 in immune regulation and autoimmunity. Immunol Cell Biol. 2012;90(1):6-13.

6 Haigis MC, Sinclair DA. Mammalian sirtuins: biological insights and disease relevance. Annu Rev Pathol. 2010;5:253-95.

7 Gillum MP, Erion DM, Shulman GI. Sirtuin-1 regulation of mammalian metabolism. Trends Mol Med. 2011;17(1):8-13.

8 Zhang J, Lee SM, Shannon S, Gao B, Chen W, Chen A, et al. The type III histone deacetylase Sirt1 is essential for maintenance of T cell tolerance in mice. J Clin Invest. 2009;119(10): 3048-58.

9 Yeung F, Hoberg JE, Ramsey CS, Keller MD, Jones DR, Frye RA, et al. Modulation of NFkappaB-dependent transcription and cell survival by the SIRT1 deacetylase. EMBO J. 2004; 23(12):2369-80.

10 Sequeira J, Boily G, Bazinet S, Saliba S, He X, Jardine $\mathrm{K}$, et al. sirt1-null mice develop an autoimmune-like condition. Exp Cell Res. 2008; 314(16):3069-74.

11 Criteria for diagnosis of Behcet's disease. International Study Group for Behçet's Disease. Lancet. 1990 May;335(8697):1078-80.
12 Engler A, Tange C, Frank-Bertoncelj M, Gay RE, Gay S, Ospelt C. Regulation and function of SIRT1 in rheumatoid arthritis synovial fibroblasts. J Mol Med. 2016;94(2):173-82.

13 Gardner PJ, Joshi L, Lee RW, Dick AD, Adamson P, Calder VL. SIRT1 activation protects against autoimmune $\mathrm{T}$ cell-driven retinal disease in mice via inhibition of IL-2/Stat5 signaling. J Autoimmun. 2013;42:117-29.

14 Hu N, Qiu X, Luo Y, Yuan J, Li Y, Lei W, et al. Abnormal histone modification patterns in lupus CD4+ T cells. J Rheumatol. 2008;35(5): 804-10.

15 Li G, Xia Z, Liu Y, Meng F, Wu X, Fang Y, et al. SIRT1 inhibits rheumatoid arthritis fibroblast-like synoviocyte aggressiveness and inflammatory response via suppressing NF- $\kappa \mathrm{B}$ pathway. Biosci Rep. 2018 Jun 21;38(3): BSR20180541.

16 Bartoli-Leonard F, Wilkinson FL, Schiro A, Inglott FS, Alexander MY, Weston R. Suppression of SIRT1 in diabetic conditions induces osteogenic differentiation of human vascular smooth muscle cells via RUNX2 signalling. Sci Rep. 2019 Jan 29;9(1):878.

17 Yao H, Sundar IK, Huang Y, Gerloff J, Sellix MT, Sime PJ, et al. Disruption of sirtuin 1-mediated control of circadian molecular clock and inflammation in chronic obstructive pulmonary disease. Am J Respir Cell Mol Biol. 2015;53(6):782-92.

18 Wu Z, Mei X, Ying Z, Sun Y, Song J, Shi W. Ultraviolet B inhibition of DNMT1 activity via $A h R$ activation dependent SIRT1 suppression in CD4+ T cells from systemic lupus erythematosus patients. J Dermatol Sci. 2017; 86(3):230-7.

19 Menssen A, Hydbring P, Kapelle K, Vervoorts J, Diebold J, Lüscher B, et al. The c-MYC oncoprotein, the NAMPT enzyme, the SIRT1inhibitor DBC1, and the SIRT1 deacetylase form a positive feedback loop. Proc Natl Acad Sci USA. 2012;109(4):E187-96.
20 Xiong S, Salazar G, Patrushev N, Alexander RW. FoxO1 mediates an autofeedback loop regulating SIRT1 expression. J Biol Chem. 2011;286(7):5289-99.

21 Sugita S, Kawazoe Y, Imai A, Kawaguchi T, Horie S, Keino H, et al. Role of IL-22- and TNF- $\alpha$-producing Th22 cells in uveitis patients with Behcet's disease. J Immunol. 2013; 190(11):5799-808.

22 Lim HW, Kang SG, Ryu JK, Schilling B, Fei M, Lee IS, et al. SIRT1 deacetylates ROR $\gamma \mathrm{t}$ and enhances Th17 cell generation. J Exp Med. 2015 May 4;212(5):607-17.

23 Yang H, Lee SM, Gao B, Zhang J, Fang D. Histone deacetylase sirtuin 1 deacetylates IRF1 protein and programs dendritic cells to control Th17 protein differentiation during autoimmune inflammation. J Biol Chem. 2013; 288(52):37256-66.

24 Donato AJ, Magerko KA, Lawson BR, Durrant JR, Lesniewski LA, Seals DR. SIRT-1 and vascular endothelial dysfunction with ageing in mice and humans. J Physiol (Lond). 2011; 589(Pt 18):4545-54.

25 Tong C, Morrison A, Mattison S, Qian S, Bryniarski M, Rankin B, et al. Impaired SIRT1 nucleocytoplasmic shuttling in the senescent heart during ischemic stress. FASEB J. 2013; 27(11):4332-42.

26 Yang SR, Wright J, Bauter M, Seweryniak K, Kode A, Rahman I. Sirtuin regulates cigarette smoke-induced proinflammatory mediator release via RelA/p65 NF-kappaB in macrophages in vitro and in rat lungs in vivo: implications for chronic inflammation and aging. Am J Physiol Lung Cell Mol Physiol. 2007; 292(2):L567-76

27 Rajendrasozhan S, Yang SR, Kinnula VL, Rahman I. SIRT1, an antiinflammatory and antiaging protein, is decreased in lungs of patients with chronic obstructive pulmonary disease. Am J Respir Crit Care Med. 2008; 177(8):861-70. 